\title{
ON A RESULT OF WINKLER
}

\section{SAKARI TOPPILA}

\section{Introduction and results}

I thank Professor O. Lehto for suggesting this subject to me.

We shall use the usual notations of the Nevanlinna theory.

Let $a_{k}$ be a sequence of non-zero complex numbers such that $a_{k} \rightarrow \infty$ as $k \rightarrow \infty$. We denote by $n(r)$ the number of points $a_{k}$ satisfying $\left|a_{k}\right| \leqq r$. It is well known that there exists an entire function of the form

$$
F(z)=\prod_{k=1}^{\infty} E_{p_{k}}\left(z / a_{k}\right),
$$

where

$$
E_{p_{k}}(u)=(1-u) \exp \left(u+\left(\frac{1}{2}\right) u^{2}+\ldots+\left(1 / p_{k}\right) u^{p_{k}}\right),
$$

such that $F$ has exactly the zeros $a_{k}$.

Let $[x]$ be the integer part of a non-negative real number $x$. Winkler [2] proved the following theorem.

Theorem A. Let $a_{k}$ be as above and suppose that $\sigma>1$. Then the entire function $F$ of the form (1.1) with

satisfies

$$
p_{k}=\left[\log n^{\sigma}\left(\left|a_{k}\right|\right)\right]
$$

$$
\log M(r, F)=O\left(N(\gamma r, 0, F)^{\sigma(1+\log r)}\right) \quad(r \rightarrow \infty)
$$

for any $\gamma>\exp (\sqrt{1 / \sigma})$.

There arise the following two questions. Does the rapid growth of $n(r)$ imply that we must take a rapidly growing sequence $p_{k}$ in (1.1), and does the rapid growth of $n(r)$ imply that, for any entire function $F$ of the form $(1.1), \log M(r, f)$ is essentially larger than $N(r, 0, f)$ ?

We shall give a negative answer to both of these questions. We prove the following

Theorem. Given any increasing function $\varphi(r)$ such that $\varphi(r) \rightarrow \infty$ as $r \rightarrow \infty$, there exists a sequence $a_{k}, a_{k} \rightarrow \infty$ as $k \rightarrow \infty$, such that the product

$$
\prod_{k=1}^{\infty}\left(1-z / a_{k}\right)
$$


converges uniformly on bounded subsets of the complex plane and that the entire function

satisfies

$$
f(z)=\prod_{k=1}^{\infty}\left(1-z / a_{k}\right)
$$

$$
\varphi(r)=O(n(r, 0, f)) \quad(r \rightarrow \infty)
$$

and

$$
\log M(r, f)=N(r, 0, f)+O(1) \quad(r \rightarrow \infty) .
$$

\section{Proof of the Theorem}

Let $z_{n}$ be the following sequence constructed by Erdös [1, Problem 4.1]: Let $z_{1}=1, z_{2}=-1$, and if $z_{n}$ has already been defined for $1 \leqq p \leqq 2^{k}$, then we define for $1 \leqq p \leqq 2^{k}$,

$$
z_{p+2^{k}}=z_{p} \exp \left(2^{-k} \pi i\right)
$$

Lemma. Let $z_{n}$ be as above, $0<d<1 / 8$, and suppose that $s \geqq 1$ is an integer. Then

$$
\left|\log \prod_{n=1}^{s}\left(1-z / z_{n}\right)\right| \leqq 4 d
$$

on $|z| \leqq d$, where $\log w$ is chosen so that $\log 1=0$.

Proof. It follows from the choice of $z_{n}$ that for any integers $p \geqq 0$ and $k \geqq 1$ there exists a real $\varphi$ such that

$$
\prod_{n=p 2^{k}+1}^{(p+1) 2^{k}}\left(1-z / z_{n}\right)=1-\left(z e^{i \varphi}\right)^{2^{k}}
$$

and that $\varphi=0$ if $p=0$.

Let $k$ be chosen so that $2^{k} \leqq s<2^{k+1}$. Then

$$
s=\sum_{p=0}^{k} t_{p} 2^{k-p},
$$

where $t_{p}\left(1-t_{p}\right)=0$ for any $p$, and we deduce from (2.2) that if $|z| \leqq d$, then

$$
\left|\log \prod_{n=1}^{s}\left(1-z / z_{n}\right)\right| \leqq 2 \sum_{p=0}^{k} t_{p} d^{2^{k-p}} \leqq 2 \sum_{p=1}^{\infty} d^{p}<4 d,
$$

which proves the Lemma.

Proof of the Theorem. Let $\varphi(r)$ be as in the Theorem. We choose a positive integer $k_{1}$ such that

and set

$$
n_{1}=2^{k_{1}}>\varphi(16)
$$

$$
a_{n}=4 z_{n} \text { for } n=1,2, \ldots, n_{1} \text {, }
$$


and if $a_{n}$ has already been defined for $n=1, \ldots, n_{p-1}$, we choose a positive integer $k_{p}$ such that

$$
n_{p}=n_{p-1}+2^{k_{p}}>\varphi\left(4^{p+1}\right)
$$

and set

Let

$$
a_{n}=4^{p} z_{n-n_{p-1}} \text { for } n=n_{p-1}+1, \ldots, n_{p} .
$$

and

$$
f_{s}(z)=\prod_{p=1}^{s}\left(1-\left(4^{-p} z\right)^{2^{k} p}\right)
$$

$$
f(z)=\lim _{s \rightarrow \infty} f_{s}(z) .
$$

Clearly $f_{s}(z) \rightarrow f(z)$ uniformly on bounded subsets of the complex plane. From (2.3) it follows that $f$ satisfies (1.3).

We define

$$
f_{s}(z)=\prod_{n=1}^{n_{s}}\left(1-z / a_{n}\right)
$$

for any $s$, and we deduce from the Lemma that if $|z| \leqq M$ and $n_{s}<t \leqq n_{s+1}$, then

$$
\begin{gathered}
\left|\log \left((1 / f(z)) \prod_{n=1}^{t}\left(1-z / a_{n}\right)\right)\right| \leqq\left|\log \left(f_{s}(z) / f(z)\right)\right|+\left|\log \prod_{n=n_{s}+1}^{t}\left(1-z / a_{n}\right)\right| \\
\leqq o(1)+O\left(M / 4^{s}\right)=o(1) \quad(t \rightarrow \infty),
\end{gathered}
$$

which implies that

$$
\prod_{n=1}^{t}\left(1-z / a_{n}\right) \rightarrow f(z)
$$

uniformly on bounded subsets of the complex plane.

Suppose that $4^{s} / 2 \leqq r<4^{s+1} / 2$. We get

$$
\begin{aligned}
& \log M(r, f)-N(r, 0, f) \leqq \sum_{q=1}^{\infty} \log \left(1+\left(r / 4^{q}\right)^{2^{k_{q}}}\right)-\sum_{q=1}^{s} 2^{k_{q}} \log ^{+}\left(r / 4^{q}\right) \\
& \leqq \sum_{q=1}^{s-1} \log \left(1+\left(4^{q} / r\right)^{2^{k_{q}}}\right)+\log \left(1+\left(r / 4^{s}\right)^{2^{k_{s}}}\right)-2^{k_{s}} \log ^{+}\left(r / 4^{s}\right)+O(1) \\
& =O(1)+\min \left\{\log \left(1+\left(r / 4^{s}\right)^{2^{k_{s}}}\right), \log \left(1+\left(4^{s} / r\right)^{2^{k_{s}}}\right)\right\}=O(1) \quad(r \rightarrow \infty) .
\end{aligned}
$$

This implies that

$$
\log M(r, f) \leqq N(r, 0, f)+O(1) \quad(r \rightarrow \infty) .
$$

From the first main theorem of the Nevanlinna theory we deduce that

$$
N(r, 0, f) \leqq T(r, f)+O(1) \leqq \log M(r, f)+O(1) \quad(r \rightarrow \infty),
$$

which together with (2.4) proves (1.4). The Theorem is proved. 


\section{References}

[1] Hayman, W. K.: Research problems in function theory. - The Athlone Press, University of London, 1967.

[2] WinkleR, J.: Über minimale Maximalbeträge kanonischer Weierstrassprodukte unendlicher Ordnung. - Resultate Math. 4, 1980, 102-116.

University of Helsinki

Department of Mathematics

SF-00100 Helsinki 10

Finland

Received 13 October 1983 